Mayr-Harting, A. (1952). J. gen. Microbiol. 7, 382-396.

\title{
The Phage Typing of Shigella sonnei, and the Limits of Type Stability
}

\author{
By ANNA MAYR-HARTING \\ Department of Bacteriology, University of Bristol
}

SUMMARY: 560 strains of Shigella sonnei were typed by Hammarström's method using bacteriophages, and the results tabulated by districts and outbreaks. Experimentally it was found that by the action of different phages transformation to a more resistant type could be achieved without conferring lysogenicity. The mechanism of this transformation seems to be in most cases the selection by the phage of true mutant types of which several are present in small numbers in every Sonne strain. The mutant types obtained most frequently out of type 3 cultures by experimental methods were also found in association with type 3 outbreaks. As dysentery phages are present in the intestines of many people, it is postulated that such transformations occur in vivo and that difference of type does not mean lack of recent genetic relationship.

Cooper \& Mayr-Harting (1951), who made a preliminary survey of the epidemiology of Sonne dysentery in Bristol, by the phage-type method, stated that 'much remains to be done to establish the degree of stability of the types in vivo and in vitro'. The present investigation falls naturally into two parts: (1) an examination of type stability in the laboratory, and (2) a further investigation into the epidemiological significance of these types.

Phage-typing of certain organisms has become firmly established as a method of epidemiology, but the results are not easily checked because the method is used by only a few people. The results are usually accepted as an absolutely reliable answer to the inquiry for the pathways of an outbreak. It is, therefore, of some use to examine the development of this method and the technique, before trying to interpret our own typing results.

Phage-typing became a possibility after Burnet (1927) showed for the first time that sensitivity to certain antibodies was parallel to sensitivity to certain bacteriophages. Later Sonnenschein (1928) used specific typhoid and paratyphoid phages, not to replace the serological examination or to complement it, but to speed up the diagnosis, as a positive result could be obtained after $4 \mathrm{hr}$. Phages were soon used extensively in Germany (Schmidt, 1932b) for quick diagnosis, but they were not used to differentiate diverse types which could not be diagnosed by serological means.

Sertic \& Boulgakov (1936a) described phages to which only typhoid strains containing the Vi antigen were sensitive, and later in the same year Scholtens (1936) and Craigie (1936) obtained results which led to the same conclusions.

Most phages investigated had their point of attack at a somatic antigen, but there are a few reports of phages which attack at flagellar antigens. Sertic \& Boulgakov (1936 $b$ ) described a phage lysing flagellated typhoid bacilli, inactive on non-motile variants. And Sievers (1943) reported the 
finding of the new salmonella type 'Köln' by its reaction to a phage which must have been flagellar. The strain in question gave the serological reactions of Salmonella typhimurium but was resistant to the Salm. typhimurium phage, subsequently it was found serologically to differ in its specific phase from Salm. typhimurium. The Breslau phage used by Schmidt (1932a) seems to have been a flagellar phage too. These phages of Schmidt's were 'adapted', i.e. he used Sonnenschein's paratyphoid phage and adapted it by numerous subcultures to other salmonella types as required.

It was observed by Craigie \& Yen (1938) that a phage attacking the typhoid $\mathrm{Vi}$ antigen could be narrowed down in specificity to certain strains, and that this phage by a process of adaptation could be made to pick out specifically other strains. All strains picked out by one such adapted phage were assumed to be of one type. This adaptation was not an absolute one, i.e. only at certain dilutions of the phage could differences be observed.

However, a subdivision of typhoid strains could thus be achieved, and this method was put to practical use by Felix (1943). Felix \& Callow (1943) extended the method to the typing of Salm. paratyphi $B$, adapting paratyphoid $\mathbf{B}$ phages in the same way.

Wilson \& Atkinson (1945), in the phage typing of staphylococci, used seven phages derived from various strains of staphylococci; eleven further phages were developed by growing one of the original phages in the presence of a strain to which it was to be adapted 'in much the same way as with Craigie's typhoid phages'. 'The adaptation into typing phages each reacting with only one type of organism could not be completely achieved. Of their fourteen types, types 1, 2 and 3 are really groups of subtypes each reacting with one or more of a group of phages. Similarly, each phage of that group reacts with one or more subtypes within a type. These subtypes are recognizable by a certain pattern of phage sensitivity, and not by sensitivity to one phage.

Five other species for which phage typing has been studied consist each of only one group of subtypes. These are Salm. typhimurium (Lilleengen, 1948) the lactic streptococci (Nichols \& Hoyle, 1949), Sonne's bacillus (Hammarström, 1949), Salm. thompson (Williams Smith, 1951a) and Salm. dublin (Williams Smith, 1951b).

Of these the phage typing of dysentery has been described in the greatest detail by Hammarström, who used twelve phages. His phage XII acts on all Sonne strains that are in the particular phase in which they are suitable for typing; Hammarström calls this the $\mathbf{R}$ phase, which seems to correspond with the phase II described by Wheeler \& Mickle (1945). His eleven other phages differ qualitatively and quantitatively, so that a very large number of reaction patterns is possible. The quantitative differences make it necessary to use each phage in a 'critical test dilution'; only so can the characteristic pattern be obtained.

Hammarström has studied technique very carefully and gives accounts of the changes of reactions brought about by the minutest changes in the composition of the nutrient medium, the drying of the plates, etc. So far, nearly a hundred types have been isolated in several thousand typed strains. 


\section{METHODS}

Organism. Rough variants are used for phage-typing of Sonne strains; fresh cultures straight from deoxycholate medium are not suitable. If subcultures on agar slopes are kept for a few days at room temperature they are found, on plating, to contain a large proportion of colonies showing the required rough morphology.

Phage. Owing to the fact that there is a critical phage concentration in which the phage must be used for the test, the phage preparations require a good deal of checking to detect slight losses of activity.

Usually it has been possible to prepare the test dilutions following Hammarström's table, but in a few cases the phages did not show the same relative activity on the standard strains; sometimes additional strains could be used to standardize the phage dilutions in these cases.

A difficulty which arose was that filtrates of strain 4 showed a marked inhibitory effect on the bacterial growth of a number of Sonne strains on agar. Eight of the twelve typing phages are propagated on strain 4 and these phage preparations, when used in fairly high concentrations, produced an inhibitory effect which could easily be mistaken for phage action where, in fact, the phage in the filtrate was inactive against the organism under test. When sufficiently diluted, this inhibitory substance ceased to affect growth at all, and did not give isolated plaques, as a phage would.

Filtrates of a broth culture prepared with the original freeze-dried strain 4 were much less inhibitory, and a new culture obtained from Dr Hammarström did not show inhibition at all.

Another irregularity was shown by phage XII, which normally produces large plaques, but when propagated with some cultures of strain 4 , it throws off small-plaque variants at a rate of approximately $98 \%$ and cannot be purified by repeated isolation from large holes. Whether these small-hole variants differ from the typical large ones in the activity of the phagecorpuscles concerned, has not been investigated.

Medium. At first $1 \frac{1}{2} \%$ agar in broth prepared from Lemco $1 \%$, peptone Oxoid $1 \%$ and $\mathrm{NaCl} 0.5 \%$ with a pH of $7 \cdot 6$, was used. Later, on Dr Hammarström's advice, $1 \%$ Difco Bacto-peptone was substituted for Oxoid peptone. Hammarström points out that the phage reactions vary a good deal with the medium, and undiluted phages showed a much greater activity on our medium than on Hammarström's. But as it was not my primary object to obtain typing results exactly comparable with Hammarström's, I retained the above medium which has the advantages of being cheap and available.

Typing. For typing, one colony is picked into nutrient broth and incubated overnight. Next morning $0.15 \mathrm{ml}$. broth culture is spread on an agar plate, the plate dried in the incubator for $\frac{1}{2}-1 \mathrm{hr}$., face up and lid removed, and then a small loopful of each of the twelve typing phages put on the plate in a definite order. The plates are read after $15 \mathrm{hr}$., not $5 \mathrm{hr}$. as in Hammarström's method.

Dr Hammarström kindly checked a large number of our typings and there were few discrepancies, but it is still possible that some of the strains which 
I typed as type 3 may be, on Hammarström's medium, less sensitive and belong to another type. On the other hand, I found that strains which Hammarström typed as type 17 , fell on our medium into two distinct groups with entirely different patterns of phage sensitivity. I have called these $17 \cdot 1$ and $\mathbf{1 7 \cdot 2}$ respectively.

\section{RESULTS}

\section{Experimental work on type stability}

This was undertaken with the purpose of gaining some idea of the stability of types; and to see whether, if the types were not stable, a trend of development from certain types to certain other types could be ascertained.

(a) Types of subcultures from individual colonies. In a number of preliminary experiments agar slope cultures of organisms of various types were plated out and ten colonies picked into broth and typed. No variation was noticed in any of these experiments, but the agar slopes were subcultures of one colony and the variation rate, if type variation existed, would have to be very high to show up in experiments involving this small order of magnitude.

(b) Types of different colonies from the same patient at one time. In another experiment ten colonies were picked up from a direct culture of faeces on desoxycholate, but these colonies, as was to be expected, were not rough, and only one of them was typical type 3 , the others showing varying degrees of resistance. From another direct culture in first isolation ten colonies were picked from desoxycholate into broth and subcultured after some days in fresh broth. These second subcultures gave typical rough flocculated growth and were all type 3 .

(c) Types of cultures from one patient at different times. The strains from some patients who gave repeated positive cultures were typed at various times. There were, in all, seventeen cases in which repeat typings were carried out, eleven were type 3 , one type 17 , one type 19 , one type 81 , one type 82 , one type 85 and one type 88 . In no case was a change of type observed.

(d) Effect on type of incubation with Hammarström's phages. 'The results are given in Table 1. A typical type 3 strain, 5631, was inoculated into broth to which Hammarström's phage III had been added. After 4 days incubation the culture was plated out and nine colonies picked into separate broth tubes. Three of the colonies chosen were rough and six were smooth, as it was thought that amongst these latter the chance of finding a variant would be better. However, all three rough colonies and only one smooth one turned out to be type 79; the other five were slightly less sensitive and did not correspond to any known type (see Exp. 3). Strain 5631 was incubated subsequently with all Hammarström's phages and besides type 79 (see Exps. 4, 6 and 8), type 23 (Exps. 7 and 9) and type 66 (Exp. 4) were obtained from the same type 3 strain. These types were found to be stable after several weeks storage at room temperature and a number of subcultures. In addition, phage patterns occurred not corresponding to any type so far known. The phage concentration used for selection varied from $1 / 3$ to $1 / 15$ of the undiluted filtrates, but more dilute filtrates would be quite suitable as even $1 / 15$ no longer yielded the 


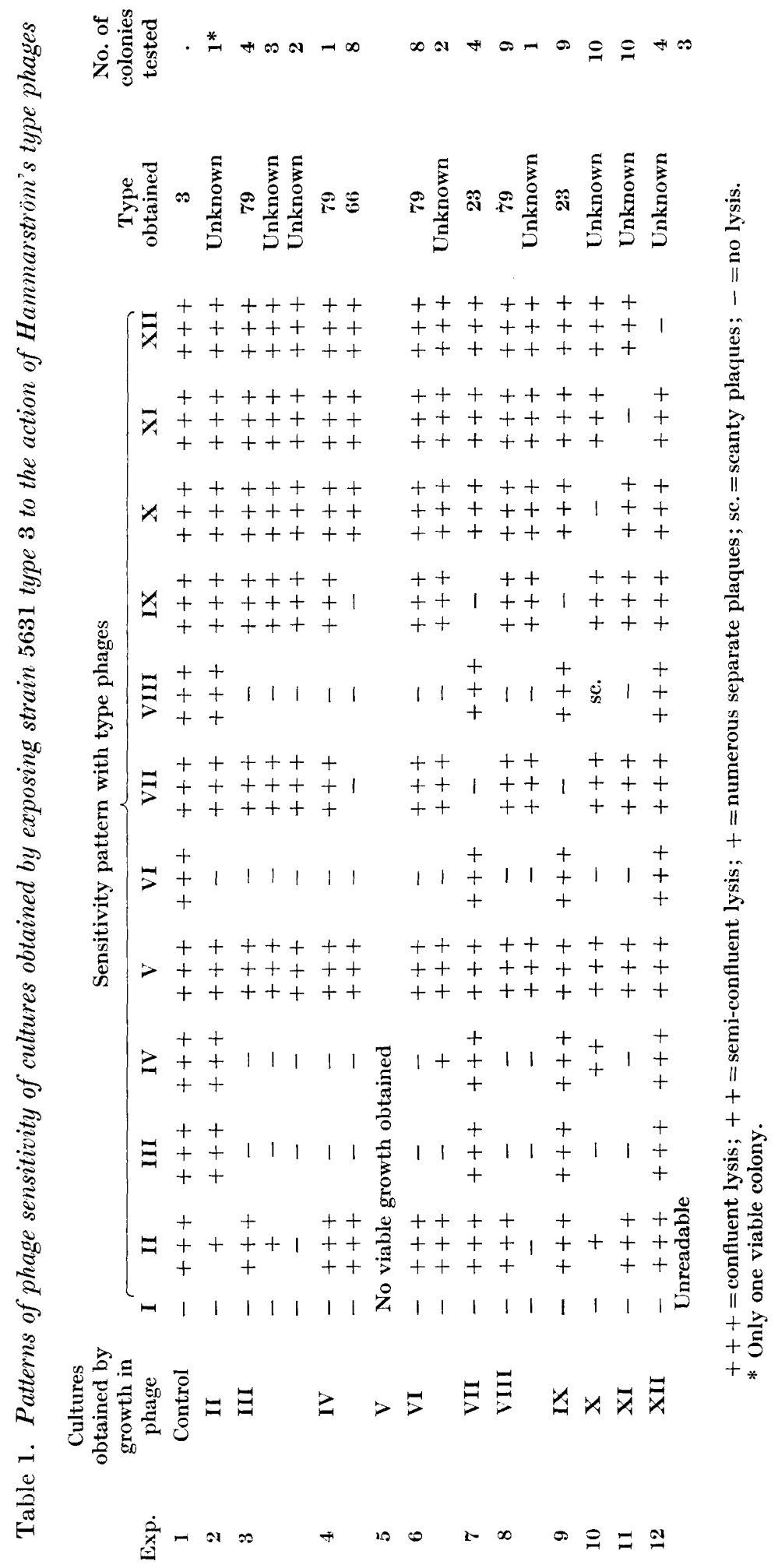


original type 3 strain and some of the more concentrated filtrates allowed only ghost colonies to grow in subculture, which on further subcultures were not viable.

(e) Effect on type of incubation with patient's phages. Transformation of types was then tried with phages isolated from the faeces of five children, suffering from Sonne dysentery.

The organisms isolated were referred to as A, B and C (type 3 ) and $\mathbf{D}$ and $\mathbf{E}$ (type 19). The corresponding filtrates from these faeces were called $a, b, c, d$ and $e$. Two of the filtrates, $a$ and $c$, were found to have slight activity against the type 19 strain $\mathrm{E}$. All other cross tests were negative.

Further experiments with filtrates $a$ and $c$. The phages present in filtrate $a$ and $c$ were each propagated by repeated incubation and filtration, with strain $\mathrm{E}$ and with strain 5631. This enhanced their activity considerably. The filtrates finally obtained were used, in the same way as the type phages previously used, to act on type 3 strain 5631 and also on type 19 strain $\mathrm{E}$, and the changed phage sensitivities of the resulting growth were noted (Tables 2-4).:

The following diagram will make clear how the filtrates were obtained and how the experiments, done with them, were connected:

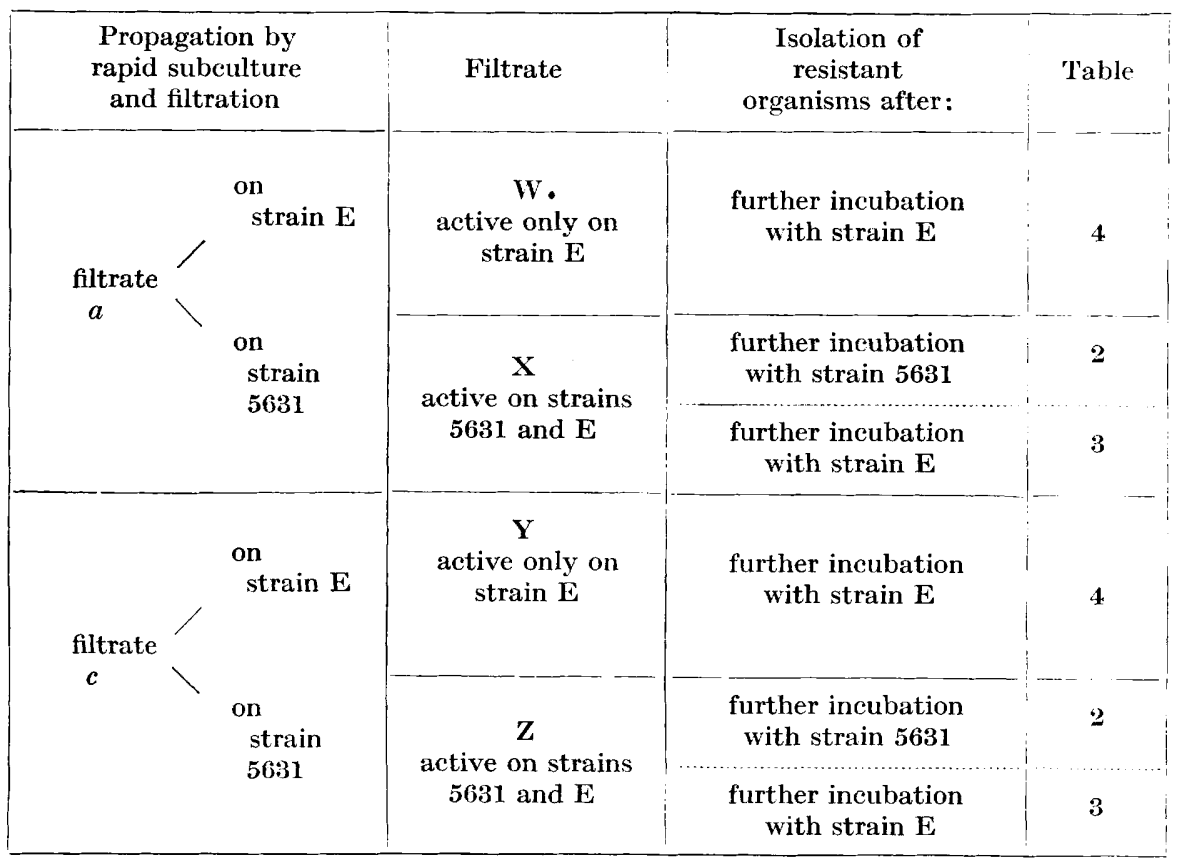

In both faeces more than one phage must have been present, as the phages propagated with strain 5631 attacked both this strain and strain E, whereas the phages propagated with strain $\mathrm{E}$ attacked strain $\mathrm{E}$ but not 5631. It is remarkable that the phages from the two faeces, both of which had been propagated with 5631 and which gave similar reactions with 5631 and $\mathrm{E}$, must 
still be different, because the transformations which they caused in type 5631 gave different new types.

None of the patterns of phage sensitivity, obtained under the influence of phages from faeces, corresponded exactly to any known type; but three out of six colonies of strain $\mathbf{5 6 3 1}$ grown in filtrate $\mathbf{X}$ gave a pattern which is at least very similar to type 79 , and the same pattern was obtained under the influence of phage VI together with typical type 79. This pattern differs from type 19 only by its additional resistance to phage VIII, and phages VI and VIII are obviously closely related (Table $\mathbf{3}$ ).

Table 2. Patterns of phage sensitivity of cultures obtained by exposing strain 5631, type 3, to the action of phages from patients' stools

(Phages propagated on strain 5631)

Patterns of phage sensitivity

\begin{tabular}{|c|c|c|c|c|c|c|c|c|c|c|c|c|c|c|}
\hline \multirow[b]{2}{*}{ Exp. } & \multirow{2}{*}{$\begin{array}{l}\text { Strain } \\
\mathbf{5 6 3 1}\end{array}$} & \multicolumn{11}{|c|}{ Patterns of phage sensitivity } & \multirow{2}{*}{$\begin{array}{c}\text { Type } \\
\text { obtained }\end{array}$} & \multirow{2}{*}{$\begin{array}{l}\text { No. of } \\
\text { colonie } \\
\text { tested }\end{array}$} \\
\hline & & I & II & III IV & V & VI & VI & & & $\mathbf{x}$ & $\Delta 1$ & III & & \\
\hline 1 & $\begin{array}{l}\text { Grown in } \\
\text { filtrate } \mathrm{X}\end{array}$ & $\begin{array}{l}- \\
-\end{array}$ & $\begin{array}{c}++ \\
+ \\
-\end{array}$ & $\begin{array}{l}\text { - sc. } \\
\text { - sc. } \\
\text { - + }\end{array}$ & $\begin{array}{l}+++ \\
+++ \\
+++\end{array}$ & $=$ & $\begin{array}{l}+++ \\
+++ \\
+++\end{array}$ & $\overline{-}$ & $\begin{array}{l}+++ \\
+++ \\
+++\end{array}$ & $\begin{array}{l}+++ \\
+++ \\
+++\end{array}$ & + & & & 2 \\
\hline 2 & $\begin{array}{l}\text { Grow } \\
\text { filtr }\end{array}$ & $\begin{array}{l}- \\
-\end{array}$ & $\begin{array}{l}- \\
-\end{array}$ & $\begin{array}{l}\text { sc. }+ \\
-\quad- \\
--\end{array}$ & - & $\begin{array}{l}- \\
-\end{array}$ & $\begin{array}{l}+ \\
+ \\
-\end{array}$ & $\begin{array}{l}- \\
-\end{array}$ & $\begin{array}{l}++ \\
++ \\
-\end{array}$ & $\begin{array}{c}++ \\
+ \\
+\end{array}$ & $\begin{array}{c}+++ \\
+++\end{array}$ & $\begin{array}{c}++ \\
++ \\
+\end{array}$ & $\begin{array}{l}\text { Unknown } \\
\text { Resistant }\end{array}$ & 2 \\
\hline
\end{tabular}

$+++=$ confluent lysis; $++=$ semi-confluent lysis; $+=$ numerous separate plaques; sc. $=$ scanty plaques; $-=$ no lysis.

\section{Tests for lysogenicity}

Workers differ in their views regarding typing strains which have acquired resistance by becoming lysogenic. Williams Smith $(1951 a, b)$ denotes the type according to the pattern which they show in the normal phage-typing test. He even goes further and assumes that certain types only arise by acquiring lysogenicity, and thereby resistance, and for a species to be suitable for phagetyping a large proportion of its strains should be carriers of different phages. Nichols \& Hoyle (1949) take the opposite view; when they find a lysogenic strain they classify it as if the strain were sensitive to the phage it carries, i.e. lysogenicity is taken as having the same significance for typing as sensitivity.

From a type 3 strain ' 8562 ', under the influence of phage IV and of phage VII, types 79, 66 and 23 were obtained. These were subcultured 6 times on Lemco agar, and then tested for lysogenicity by three different methods: (1) heating of broth cultures $\frac{1}{2} \mathrm{hr} .56^{\circ} ;(2)$ filtration of broth cultures through Chamberland filters; (3) mixed culture with the sensitive original strain and subsequent filtration. The heated culture and the filtrates were then spotted on to a confluent inoculum of the original sensitive type 3, and another freshly isolated strain, also type 3. No phage action was found by any of these methods. The three variants were checked at this stage for phage-type and found to be type 79,66 and 23 respectively. The significance of these experiments will be discussed later. 


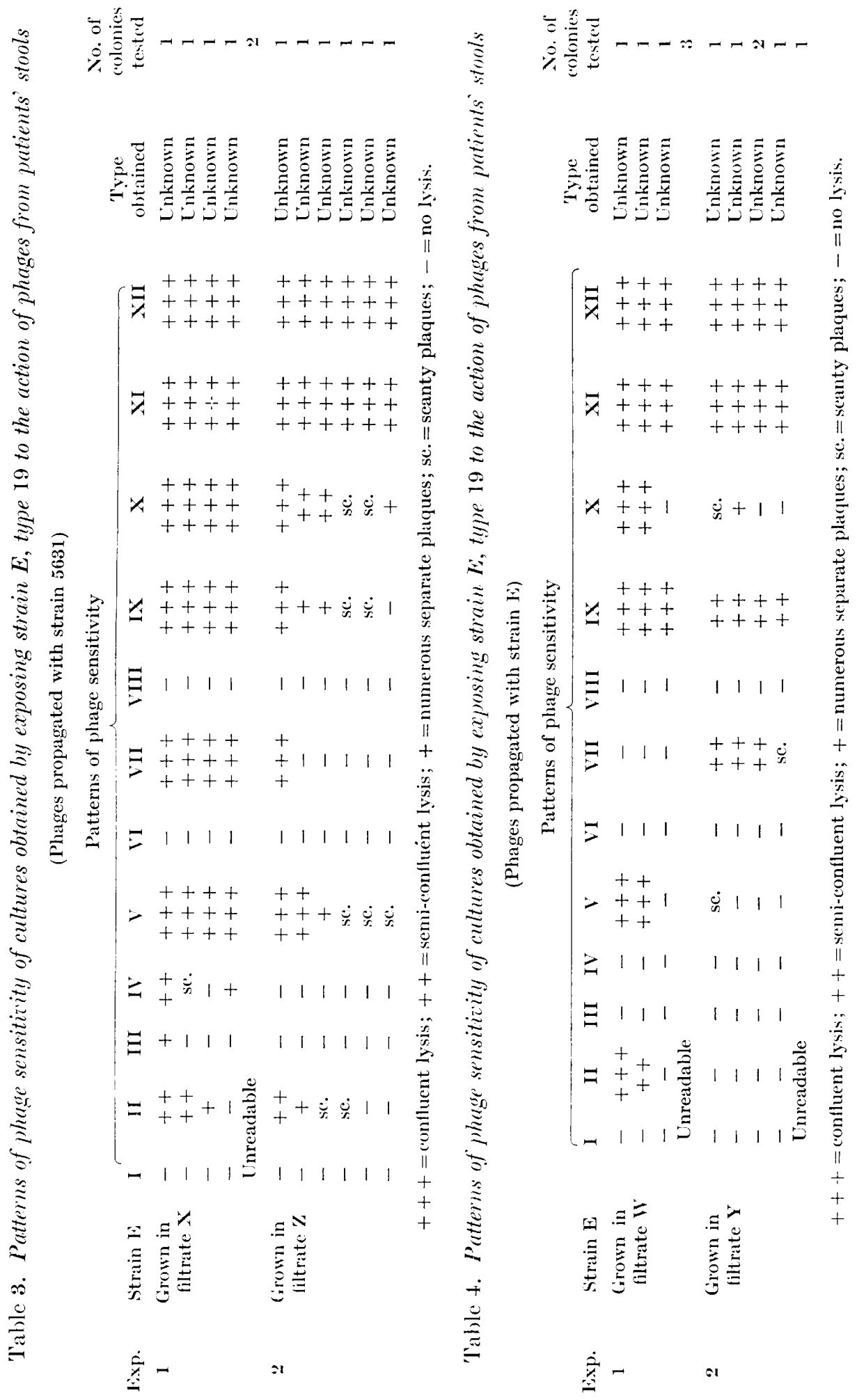




\section{Typing results of Sonne dysentery from patients}

The results of our typing are given in Table 5; 517 strains are from Bristol or the immediately adjoining districts, 43 are from other parts of the country; $455(81 \cdot 25 \%)$ out of 560 strains were type 3 . In Sweden, type 3 is the second most frequent with $23 \%$, and in Czechoslovakia, where Raška, Mališová \& Mazáček (1950) did Sonne phage-typing, with Hammarström's phages, it is with $\mathbf{2 9 . 7} \%$ the most frequent type. Where the number of strains investigated is sufficiently large, the distribution of types throughout Bristol city is not very different. A number of types occurred only once or twice. Of these, $12 a$ was isolated from a boy who fell ill during his return journey from a French holiday; Dr Hammarström informed us that all his strains of $12 a$, without exception, came from France. We isolated three strains of type 66; two of these were isolated from patients, unrelated to each other, who had just been to France. The third strain of this type was obtained from a little girl who was found at the same time to carry Salm. paratyphi B, when at that time no other cases were known to exist in Bristol.

The predominant type in most outbreaks was type 3 and in a number of outbreaks this was the only type found.

\section{Outbreaks of one type}

Outbreak I. In a colony for mentally defective people thirty-six cases of type 3 occurred over a period of 1 year. No other type was found and it is assumed that all these infections were acquired at the colony.

Outbreaks $I I-I V$ involved twenty-two, seven and nineteen people respectively and were due to type 3 .

Outbreaks $V I I I-X$ were small outbreaks in one hospital occurring over a period of 1 year.

Outbreak VIII was due to type 81. This type was for a short time found in a number of isolated cases. One of these was transferred to hospital and within a few days there was an outbreak in another ward involving about a dozen people. Nine of their cultures were typed as 81 . No proof of the method of transfer has been obtained, but a member of the staff subsequently gave a history of intestinal upset. Strains of type 81 were not isolated after this outbreak.

Outbreak $I X$ was only a very small outbreak. After the introduction of a case of type 3 , three cases in the same ward, all close contacts, acquired the infection.

Outbreak $X$ was rather involved, but it seems that it consisted of six cases of type 88 and that a few cases of type 21 and type 3 that were in hospital at the same time were independent of that outbreak and of one another.

\section{Outbreaks of more than one type}

Outbreak $V$. This involved fourteen people at a day nursery. Thirteen of the strains were type 3 , one was type 85 .

Outbreak VI and VII. These were large outbreaks at mental hospitals, one of fifty-five, the other of forty-one cases. The first yielded fifty-one cultures 

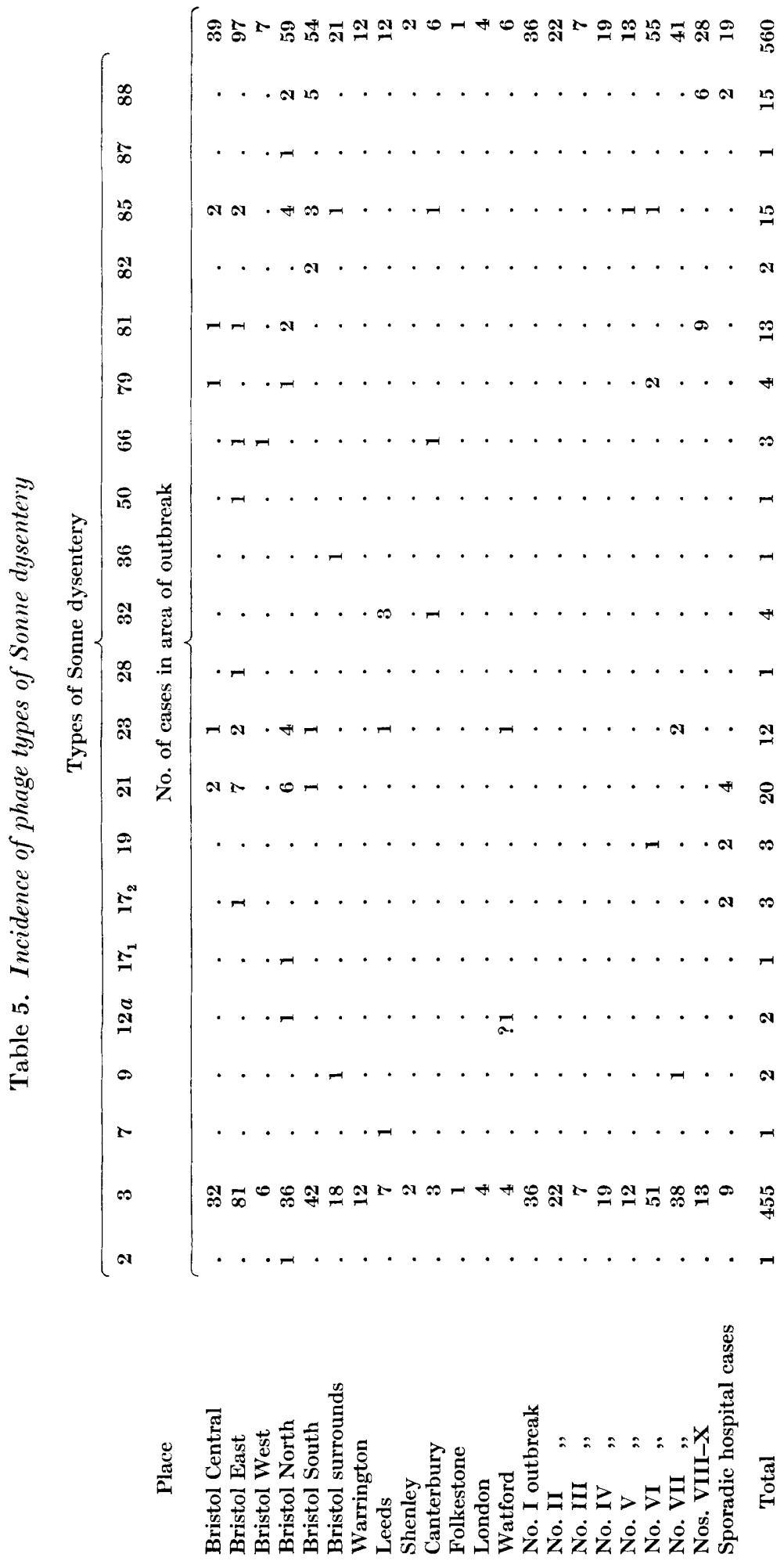
of type 3 , one patient had type 19, one type 85 and two type 79 ; from the other outbreak we obtained thirty-eight strains of type 3 , two of type 23 , one of type 9 .

\section{INTERPRETATION OF TYPING RESULTS AND GENERAL DISCUSSION}

When Craigie \& Yen first used their type classification of typhoid strains for epidemiological purposes they were well aware that it stood or fell with the stability of type. They did not obtain any experimental evidence of change of type in multiple isolations from one patient, nor in multiple colony isolations

Table 6. Phage sensitivity pattern of strains found in outbreaks together with type 3

\begin{tabular}{|c|c|c|c|c|c|c|c|c|c|c|c|c|}
\hline \multirow[b]{2}{*}{ Type } & \multicolumn{12}{|c|}{ Phage } \\
\hline & I & II & III & IV & V & VI & VII & VIII & IX & $\mathbf{x}$ & XI & XII \\
\hline $\begin{array}{r}3 \\
85 \\
19 \\
79 \\
9 \\
\mathbf{2 3}\end{array}$ & $\begin{array}{l}- \\
- \\
- \\
- \\
-\end{array}$ & $\begin{array}{l}+++ \\
+++ \\
+++ \\
+++ \\
+++ \\
+++\end{array}$ & $\begin{array}{c}+++ \\
- \\
- \\
- \\
+++ \\
+++\end{array}$ & $\begin{array}{c}+++ \\
+++ \\
+++ \\
- \\
+++ \\
+++\end{array}$ & $\begin{array}{l}+++ \\
+++ \\
+++ \\
+++ \\
+++ \\
+++\end{array}$ & $\begin{array}{c}+++ \\
+ \\
- \\
- \\
+++ \\
+++\end{array}$ & $\begin{array}{c}+++ \\
+++ \\
+++ \\
+++ \\
- \\
-\end{array}$ & $\begin{array}{c}+++ \\
+++ \\
+++ \\
- \\
+++ \\
+++\end{array}$ & $\begin{array}{c}+++ \\
+++ \\
+++ \\
+++ \\
+++ \\
+\end{array}$ & $\begin{array}{l}+++ \\
+++ \\
+++ \\
+++ \\
+++ \\
+++\end{array}$ & $\begin{array}{l}+++ \\
+++ \\
+++ \\
+++ \\
+++ \\
+++\end{array}$ & $\begin{array}{l}+++ \\
+++ \\
+++ \\
+++ \\
+++ \\
+++\end{array}$ \\
\hline
\end{tabular}

from the same culture, but they admitted that the natural transfer in man would offer greater opportunities. They say 'the isolation of different types of $B$. typhosus from apparently related cases would appear to demand further investigation since such a discrepancy would indicate that either the epidemiological information or the concept of type stability was erroneous'. In spite of this cautious dictum they were rather inclined to assume the stability of types. There has been a marked tendency lately to abandon even the caution and reserve that Craigie \& Yen had expressed.

Felix (1943) states that the phage type of a strain is for all practical purposes a permanent character; a disquieting discrepancy is explained as an 'accident during the handling of the cultures'. Only in certain specified instances are changes of type admitted to occur (Craigie \& Felix, 1947; Anderson, 1951).

Hammarström also has observed outbreaks in which a change of type occurred, and even changes of type in one patient. Some of these might indicate the introduction of a new strain but some he admits to be real changes of type.

In our material there are several cases where different types have occurred in seemingly related cases: (1) the outbreaks, V-VII, each with one or more cases of types other than the predominant type $3 ;(2)$ the very striking case of two brothers, one with type 3 , the other with type 23 ; (3) two students in a training college, one type 3 , and the other type 85 . Only in the last case do the epidemiological data support the assumption that no genetical relationship exists between the two strains. In the outbreak $\mathrm{V}$ outside contacts existed so that the introduction of other types could not be excluded. In the other 
outbreaks, particularly in the example of the two brothers, a genetic relationship is likely although, owing to the widespread incidence of Sonne dysentery at the time, the introduction of other types cannot be excluded with certainty, nor is it certain that a mixed infection was not acquired, and different types survived in different people.

From the outbreak VI, two strains of type 79, one strain of type 85 and one of type 19 were obtained in an otherwise type 3 outbreak. In my experimental work I have shown that the transformation of type 3 to type 79 can be achieved by a number of different phages. Transformation of type 19, a type which also occurred in this outbreak, to a type closely resembling type 79 was actually achieved experimentally using a filtrate of a patient's faeces. Type 85 is only very slightly different from type 19 . There is no doubt, therefore, that the four types that occurred in that outbreak have close genetic links and that the opportunity for the transformation existed in vivo.

In outbreak VII, two strains of type 23 and one of type 9 occurred; these are closely related to one another and type 3 is easily transformed into type $\mathbf{2 3}$ by the action of Hammarström's phages VII or IX. This transformation may also have taken place in the case of the two brothers with types 3 and 23.

In one experiment one phage, Hammarström's phage IV, acting on a type 3 culture yielded two different types, 79 and 66 . It is reasonable therefore to assume some genetic relationship between these three types. Table 6 shows the phage sensitivity patterns of the strains concerned.

If the transformation of types by phages occurs in the field, any unusual type isolated may have one of two sources: $(a)$ a carrier of that type; $(b)$ a carrier of a bacteriophage which can transform a more common type into the one in question. One case reported by Felix (1943) can be xplained on these lines. A new typhoid type D5 was isolated from an Indian soldier who fell ill several months after his arrival in this country. Felix interprets this as the strain having been imported from India, but it is possible that the man imported a particular bacteriophage, became infected with a $\mathbf{D}$ strain in this country, and the transformation to D 5 was effected by the bacteriophage.

Similarly, the patients who fell ill with type 66 dysentery after their return from France may have picked up the strain there, or they may have picked up a phage, perhaps common in France, which transformed an ordinary type 3 strain into type 66 .

In an epidemiological investigation phage typing of strains alone may be misleading. Where, in an outbreak, different phage types are encountered, only an analysis of the pathways through patients and carriers of the bacteriophages as well as those of the infecting strains will provide the true story; a truly formidable task.

Nicolle and his co-workers $(1951 a-c)$ found that the phage types of Salm. paratyphi $\mathrm{B}$ were largely determined by their lysogenic properties, and most naturally occurring types could be produced artificially by infecting an appropriate basic type with the appropriate $\mathrm{Vi}$ phage. The three basic types $\mathbf{1}$, $3 a$ and $3 b$, could not, on the other hand, be transformed into one another by means of the Vi phages which they carried. Nicolle concludes, therefore, that 
lysogenicity is not the only factor that determines the phage type. He suggests that the lysogenic phage types may have arisen by contamination of the basic types with phages encountered in the intestine.

Williams Smith $(1951 a, b)$, using 0 phages for the typing of Salm. thompson and Salm. dublin had similar results. Some phage types were dependent on acquired resistance to a certain phage, others not. Some of his strains of Salm. dublin, made resistant to two phages, were found to carry only one. This leads to the conclusion that in that group of organisms even acquired phage resistance is not necessarily associated with lysogenicity.

The three variant types which $\mathbf{I}$ obtained from a type $\mathbf{3}$ strain were free from phage infection, and as the phage which they would have carried was known, it could not have been missed by using insensitive indicator strains. The process of change of type, in the case of Sonne dysentery, seems to be due to the presence of a number of true spontaneous mutants, far too small in numbers to show up in the usual typing test, but selected in a medium which contains a phage to which they are resistant. In support of this is the fact that the new types were resistant to other phages besides the one used for their selection, and that under the influence of one phage on one strain two types could be obtained, having in common resistance to the selecting phage, but otherwise differing in their pattern of sensitivity.

From the literature and from my own experimental results it seems that under the influence of a phage the phage type of an organism can be changed in vitro in two fundamentally different ways: (1) by acquiring lysogenicity and thereby resistance to that particular phage; $(2)$ by the selection of pre-existent mutants resistant to the phage.

We do not know much about the role which these transformations play in the field. Williams Smith (1948) has found evidence of change of type of staphylococci in infected udders due to the influence of bacteriophage. Bacteriophages are known to be present in every intestine, and opportunities for the interaction of bacteria and phages are likely to occur.

The high incidence and ubiquitousness of Sonne dysentery might still allow one to argue the occurrence of unrelated strains of different type in one outbreak. In the writer's limited experience of typhoid and paratyphoid outbreaks there are two examples where multiple phage types were found when multiple infection seemed, on epidemiological considerations, extremely unlikely.

Vi-phage types may be more stable than 0-phage types in the field. Felix (1943) pointed out that, whereas typhoid 0 -phages are very frequently found in stools, the finding of Vi phages is rather rare. This may be the reason for the stability of Vi types, as compared with Sonne types. But the Vi-type is not influenced by Vi phages only, as Anderson (1951) showed when he demonstrated that the change of type $\mathbf{F} 1 \rightarrow \mathbf{F} 2$ is dependent on an 0 -phage.

In the evaluation of phage-typing it is usual to argue from the typing results, as a priori correct, to the epidemiological information, thereby assuming an absolute stability of phage type. This assumption seems to me open to grave doubt, and much useful knowledge might be gained by accepting instability in vitro as an established fact and instability in vivo at least as a working 
hypothesis. As all findings of different phage types have hitherto been interpreted as due to different sources of infection we have no information on the incidence of change of type in vivo. Only when this is known for every organism, together with the genetic relationship between types, shall we be able to evaluate phage-typing results in a meaningful way.

I wish to thank Prof. K. E. Cooper for his continued interest and for much useful advice; Dr E. Hammarström who kindly sent us strains and phages and checked the type of numerous strains; Dr W. A. Gillespie in Bristol, Dr S. Bobra in Warrington, Dr G. M. Williamson in Leeds, and Dr G. B. Forbes in Canterbury for Sonne strains; Dr J. Macrae, Dr N. S. Craig, Dr Harris Caplan and Mr J. E. Mountjoy for epidemiological data and Mr W. L. Brawn for help with the typing.

\section{REFERENCES}

Anderson, E. S. (1951). The significance of Vi-phage types F1 and F2 of Salmonella typhi. J. Hyg., Camb. 49, 458.

BURNET, F. M. (1927). The relationship between heat-stable agglutinogens and sensitivity to bacteriophage in the salmonella group. Brit. J. exp. Path. 8, 121.

Cooper, K. E. \& Mayr-Harting, A. (1951). Phage-typing and epidemiology of Shigella dysenteriae Sonne. Brit. med. J. ii, 271.

Craigie, J. (1936). A bacteriophage for the Vi-form of Salmonella typhi. J. Bact. $31,56$.

Craigie, J. \& Yen, C. H. (1938). The demonstration of types of B. typhosus by means of preparation of type II Vi phage 1. Principles and Technique. 2. The stability and epidemiological significance of Vi-form types of B. typhosus. Canad. J. publ. Hlth, 29, 448.

Craigie, J. \& Felix, A. (1947). Typing of typhoid bacilli with Vi bacteriophage. Lancet, i, 823.

Felix, A. (1943). Experiences with typing of typhoid bacilli by means of Vi bacteriophage. Brit. med. $J$. i, 435.

Felix, A. \& Callow, B. R. (1943). Typing of paratyphoid B bacilli by means of Vi bacteriophage. Brit. med. J. ii, 127.

Hammarström, E. (1949). Phage-typing of Shigella sonnei. Acta med. scand. (Suppl.), no. 223.

Hamon, Y. \& Nicolle, P. $(1951 b)$. Recherches sur les facteurs qui conditionnent l'appartenance des bacilles paratyphiques $\mathbf{B}$ aux différents types bacteriophagiques de Felix et Callow. 2. Obtention expérimentale, à partir des cultures non lysogènes, des types de $S$. paratyphi $\mathbf{B}$ par contamination avec les bacteriophages extraits des types lysogènes. Ann. Inst. Pasteur, 80, 496.

LilleENGen, K. (1948). Typing of Salmonella typhimurium by means of bacteriophage. Acta path. microbiol. scand. (Suppl.), no. 77.

Nicolle, P., Hamon, Y. \& Edlinger, E. (1951 $a$ ). Recherches sur les facteurs qui conditionnent l'appartenance des bacilles paratyphiques $\mathbf{B}$ aux différents types bacteriophagiques de Felix et Callow. 1. La lysogénéité des différents types de Salmonella paratyphi B. Ann. Inst. Pasteur, 80, 479.

Nicolle, P. \& Hamon, Y. (1951 b). Recherches sur les facteurs qui conditionnent l'appartenance des bacilles paratyphiques $B$ aux differents types bacteriophagiques de Felix et Callow. 3. Nouvelles études sur les transformations de types par l'action des bacteriophages extraits des bacilles lysogènes. Ann. Inst. Pasteur, 81, 614.

Nichols, A. A. \& Hoyle, M. (1949). Bacteriophage in typing lactic streptococci. J. Dairy Res. 16, $16 \%$.

Raška, K., Malıšová, V., \& MazÁček, M. (1950). Praktický Význam Fagotypisace v Epidemiologii střevnich Nákaz. Čas. Lék. čes. 89, 835. 
Schmidt, A. (1932a). Gewinnung spezifisch eingestellter Diagnostikphagen durch Umzüchtung. Zbl. Bakt. (1. Abt. Orig.), 123, 202.

Schмidt, A. (1932b). Über die Verwendung des Bakteriophagen in der Typhus- und Paratyphusdiagnose. Zbl. Bakt. (1. Abt. Orig.), 123, 207.

Scholtens, R. Тн. (1936). The resistance developed against bacteriophage. J. Hyg., Camb. 36, 452.

Sertic, V. \& Boulgakov, A. N. (1936a). Sur la sensibilité des souches d'Eberthella typhi au bactériophage avec les caractères antigèniques. C.R. Soc. Biol., Paris, $122,35$.

Sertic, V. \& Boulgakov, N. A. (1936b). Bacteriophages specifiques pour des variétés bactériennes flagellées. C.R. Soc. Biol., Paris, 123, 887.

Sievers, M. (1943). Ein neuer Salmonellatyp 'Köln' durch Phagendiagnostik ermittelt. Z Zbl. Bakt. (1. Abt. Orig.), 150, 52.

Sonnenschein, C. (1928). Bakteriendiagnose mit Bakteriophagen. Dtsch. med. Wschr. 54, 1034.

Wheeler, K. M. \& Mickle, F. L. (1945). Antigens of Shigella sonnei. J. Immunol. $51,257$.

Wrlliams Smith, H. (1948). Investigations on the typing of staphylococci by means of bacteriophage. II. The significance of lysogenic strains in staphylococcal type designation. J. Hyg., Camb. 46, 82.

Williams Smith, H. (1951 $a)$. The typing of Salmonella thompson by means of bacteriophage. J. gen. Microbiol. 5, 472.

Williams Smith, H. (1951b). The typing of Salmonella dublin by means of bacteriophage. J. gen. Microbiol. 5, 919.

Wilson, G. S. \& Atrinson, J. D. (1945). Typing of staphylococei by the bacteriophage method. Lancet, i, 647.

(Received 21 May 1952) 